Mathematical Modelling and Analysis

Volume 21 Number 6, November 2016, 836-851

http://dx.doi.org/10.3846/13926292.2016.1247755

(C) Vilnius Gediminas Technical University, 2016
Publisher: Taylor\&Francis and VGTU

http://www.tandfonline.com/TMMA

ISSN: $1392-6292$

eISSN: $1648-3510$

\title{
Integral Error Representation of Hermite Interpolating Polynomial and Related Inequalities for Quadrature Formulae
}

\author{
Gorana Aras-Gazić ${ }^{a}$, Josip Pečarić ${ }^{b}$ and Ana Vukelićc \\ ${ }^{a}$ Faculty of Architecture, University of Zagreb \\ Fra Andrije Kacica Miosica 26, 10000 Zagreb, Croatia \\ ${ }^{b}$ Faculty of Textile Technology, University of Zagreb \\ Prilaz baruna Filipovica 28a, 10000 Zagreb, Croatia \\ ${ }^{c}$ Faculty of Food Technology and Biotechnology, Mathematics department, \\ University of Zagreb \\ Pierottijeva 6, 10000 Zagreb, Croatia \\ E-mail(corresp.): avukelic@pbf.hr \\ E-mail: garas@arhitekt.hr \\ E-mail: pecaric@element.hr
}

Received March 8, 2016; revised October 29, 2016; published online November 15, 2016

\begin{abstract}
We consider integral error representation related to the Hermite interpolating polynomial and derive some new estimations of the remainder in quadrature formulae of Hermite type, using Hölder's inequality and some inequalities for the Čebyšev functional. As a special case, generalizations for the zeros of orthogonal polynomials are considered.

Keywords: Hermite interpolating polynomial, Green function, quadrature formulae, Hölder's inequality, Čebyšev functional, orthogonal polynomials.
\end{abstract}

AMS Subject Classification: 26D15; 26D07; $26 \mathrm{~A} 51$.

\section{Introduction}

We follow here notations and terminology about Hermite interpolating polynomial from [1, p. 62]. Let $-\infty<a<b<\infty$ and $a \leq a_{1}<a_{2} \ldots<a_{r} \leq b$, $(r \geq 2)$ be given. For $f \in C^{n}[a, b]$ a unique polynomial $P_{H}(t)$ of degree $(n-1)$, exists, fulfilling one of the following conditions: Hermite conditions:

$$
P_{H}^{(i)}\left(a_{j}\right)=f^{(i)}\left(a_{j}\right) ; \quad 0 \leq i \leq k_{j}, \quad 1 \leq j \leq r, \quad \sum_{j=1}^{r} k_{j}+r=n,
$$

in particular, simple Hermite or Osculatory conditions, $n=2 m, r=m, k_{j}=1$ for all $j$ :

$$
P_{O}\left(a_{j}\right)=f\left(a_{j}\right), \quad P_{O}^{\prime}\left(a_{j}\right)=f^{\prime}\left(a_{j}\right), \quad 1 \leq j \leq m
$$


Lagrange conditions, $r=n, k_{j}=0$ for all $j$ :

$$
P_{L}\left(a_{j}\right)=f\left(a_{j}\right), \quad 1 \leq j \leq n,
$$

type $(m, n-m)$ conditions, $r=2,1 \leq m \leq n-1, k_{1}=m-1, k_{2}=n-m-1$ :

$$
\begin{aligned}
& P_{m n}^{(i)}(a)=f^{(i)}(a), \quad 0 \leq i \leq m-1, \\
& P_{m n}^{(i)}(b)=f^{(i)}(b), \quad 0 \leq i \leq n-m-1,
\end{aligned}
$$

two-point Taylor conditions, $n=2 m, r=2, k_{1}=k_{2}=m-1$ :

$$
P_{2 T}^{(i)}(a)=f^{(i)}(a), P_{2 T}^{(i)}(b)=f^{(i)}(b), \quad 0 \leq i \leq m-1 .
$$

The associated error $\left|e_{H}(t)\right|$ can be represented in terms of the Green's function $G_{H}(t, s)$ for the multipoint boundary value problem

$$
z^{(n)}(t)=0, \quad z^{(i)}\left(a_{j}\right)=0, \quad 0 \leq i \leq k_{j}, \quad 1 \leq j \leq r,
$$

that is, the following result holds [1]:

Theorem 1. Let $F \in C^{n}[a, b]$ and let $P_{H}$ be its Hermite interpolating polynomial. Then

$$
F(t)=P_{H}(t)+e_{H}(t)=\sum_{j=1}^{r} \sum_{i=0}^{k_{j}} H_{i j}(t) F^{(i)}\left(a_{j}\right)+\int_{a}^{b} G_{H}(t, s) F^{(n)}(s) d s,
$$

where $H_{i j}$ are fundamental polynomials of the Hermite basis defined by

$$
H_{i j}(t)=\frac{1}{i !} \frac{\omega(t)}{\left(t-a_{j}\right)^{k_{j}+1-i}} \sum_{k=0}^{k_{j}-i} \frac{1}{k_{j} !}\left[\frac{\left(t-a_{j}\right)^{k_{j}+1}}{\omega(t)}\right]_{t=a_{j}}^{(k)}\left(t-a_{j}\right)^{k},
$$

where $\omega(t)=\prod_{j=1}^{r}\left(t-a_{j}\right)^{k_{j}+1}$ and $G_{H}$ is the Green's function defined by

$$
G_{H}(t, s)=\left\{\begin{array}{l}
\sum_{j=1}^{l} \sum_{i=0}^{k_{j}} \frac{\left(a_{j}-s\right)^{n-i-1}}{(n-i-1) !} H_{i j}(t), s \leq t, \\
-\sum_{j=l+1}^{r} \sum_{i=0}^{k_{j}} \frac{\left(a_{j}-s\right)^{n-i-1}}{(n-i-1) !} H_{i j}(t), s \geq t,
\end{array}\right.
$$

for all $a_{l} \leq s \leq a_{l+1}, 0=1, \ldots, r\left(a_{0}=a, a_{r+1}=b\right)$.

In [4] M. Bessenyei and Zs. Páles considered the following Gauss type quadrature formulae where the coefficients and the base points are to be determined so that be exact when $f$ is a polynomial of degree at most $2 n-1,2 n, 2 n$ and $2 n+1$, respectively (see also [3]):

$$
\begin{aligned}
& \int_{a}^{b} \rho(t) f(t) d t=\sum_{k=1}^{n} c_{k} f\left(\xi_{k}\right), \quad \int_{a}^{b} \rho(t) f(t) d t=c_{0} f(a)+\sum_{k=1}^{n} c_{k} f\left(\xi_{k}\right), \\
& \int_{a}^{b} \rho(t) f(t) d t=\sum_{k=1}^{n} c_{k} f\left(\xi_{k}\right)+c_{n+1} f(b) \\
& \int_{a}^{b} \rho(t) f(t) d t=c_{0} f(a)+\sum_{k=1}^{n} c_{k} f\left(\xi_{k}\right)+c_{n+1} f(b) .
\end{aligned}
$$


In this paper we use integral error representation of Hermite interpolating polynomial and the above formulae to get some new estimations of the remainder in quadrature formulae of Hermite type. We will consider a special case, in which the base points turn out to be the zeros of orthogonal polynomials. We also use inequalities for the Čebyšev functional in terms of the first derivative (see [5]) for some new bounds for the remainder.

\section{Estimations of the remainder in quadrature formulae of Hermite type}

In this section we use integral error representation from Theorem 1 to prove a number of inequalities for weighted Hermite quadrature rule using $L_{p}$ norms for $1 \leq p \leq \infty$.

Theorem 2. Suppose that all assumptions of Theorem 1 hold. Assume that $\rho:[a, b] \rightarrow \mathbb{R}$ is a nonnegative integrable function with $\int_{a}^{b} \rho(t) d t>0$ and $(p, q)$ is a pair of conjugate exponents, that is $1 \leq p, q \leq \infty, 1 / p+1 / q=1$. Then we have

$$
\begin{gathered}
\left|\int_{a}^{b} \rho(t) F(t) d t-\sum_{j=1}^{r} \sum_{i=0}^{k_{j}} F^{(i)}\left(a_{j}\right) \int_{a}^{b} \rho(t) H_{i j}(t) d t\right| \\
\leq\left\|F^{(n)}\right\|_{p}\left(\int_{a}^{b}\left|\int_{a}^{b} \rho(t) G_{H}(t, s) d t\right|^{q} d s\right)^{1 / q}
\end{gathered}
$$

The constant on the right-hand side of (2.1) is sharp for $1<p \leq \infty$ and the best possible for $p=1$.

Proof. First, we multiply identity $(1.1)$ by $\rho(t)$ and then integrate on interval $[a, b]$. Let's denote

$$
\Phi(s)=\int_{a}^{b} \rho(t) G_{H}(t, s) d t .
$$

By applying Hölder's inequality we obtain

$$
\begin{gathered}
\left|\int_{a}^{b} \rho(t) F(t) d t-\sum_{j=1}^{r} \sum_{i=0}^{k_{j}} F^{(i)}\left(a_{j}\right) \int_{a}^{b} \rho(t) H_{i j}(t) d t\right| \\
=\left|\int_{a}^{b} \Phi(s) F^{(n)}(s) d s\right| \leq\left\|F^{(n)}\right\|_{p}\left(\int_{a}^{b}|\Phi(s)|^{q} d s\right)^{1 / q} .
\end{gathered}
$$

For the proof of the sharpness of the constant $\left(\int_{a}^{b}|\Phi(s)|^{q} d s\right)^{1 / q}$ let us find a function $F$ for which the equality in (2.1) is obtained. For $1<p<\infty$ take $F$ to be such that

$$
F^{(n)}(s)=\operatorname{sgn} \Phi(s)|\Phi(s)|^{\frac{1}{p-1}} .
$$

For $p=\infty$ take $F^{(n)}(s)=\operatorname{sgn} \Phi(s)$. For $p=1$ we prove that

$$
\left|\int_{a}^{b} \Phi(s) F^{(n)}(s) d s\right| \leq \max _{s \in[a, b]}|\Phi(s)|\left(\int_{a}^{b}\left|F^{(n)}(s)\right| d s\right)
$$


is the best possible inequality. Suppose that $|\Phi(s)|$ attains its maximum at $s_{0} \in[a, b]$. First we assume that $\Phi\left(s_{0}\right)>0$. For $\varepsilon$ small enough we define $F_{\varepsilon}(s)$ by

$$
F_{\varepsilon}(s)= \begin{cases}0, & a \leq s \leq s_{0}, \\ \frac{1}{\varepsilon n !}\left(s-s_{0}\right)^{n}, & s_{0} \leq s \leq s_{0}+\varepsilon \\ \frac{1}{(n-1) !}\left(s-s_{0}\right)^{n-1}, & s_{0}+\varepsilon \leq s \leq b .\end{cases}
$$

Then for $\varepsilon$ small enough

$$
\left|\int_{a}^{b} \Phi(s) F^{(n)}(s) d s\right|=\left|\int_{s_{0}}^{s_{0}+\varepsilon} \Phi(s) \frac{1}{\varepsilon} d s\right|=\frac{1}{\varepsilon} \int_{s_{0}}^{s_{0}+\varepsilon} \Phi(s) d s .
$$

Now from the inequality (2.3) we have

$$
\frac{1}{\varepsilon} \int_{s_{0}}^{s_{0}+\varepsilon} \Phi(s) d s \leq \Phi\left(s_{0}\right) \int_{s_{0}}^{s_{0}+\varepsilon} \frac{1}{\varepsilon} d s=\Phi\left(s_{0}\right) .
$$

Since

$$
\lim _{\varepsilon \rightarrow 0} \frac{1}{\varepsilon} \int_{s_{0}}^{s_{0}+\varepsilon} \Phi(s) d s=\Phi\left(s_{0}\right)
$$

the statement follows. In the case $\Phi\left(s_{0}\right)<0$, we define $F_{\varepsilon}(s)$ by

$$
F_{\varepsilon}(s)= \begin{cases}\frac{1}{(n-1) !}\left(s-s_{0}-\varepsilon\right)^{n-1}, & a \leq s \leq s_{0}, \\ -\frac{1}{\varepsilon n !}\left(s-s_{0}-\varepsilon\right)^{n}, & s_{0} \leq s \leq s_{0}+\varepsilon, \\ 0, & s_{0}+\varepsilon \leq s \leq b\end{cases}
$$

and the rest of the proof is the same as above.

Taking $n=2 m, r=m, k_{j}=1$ for all $j$ in Theorem 2 we obtain the inequalities with simple Hermite or Osculatory conditions:

Corollary 1. Let $F \in C^{2 m}([a, b]), m \geq 1$. Assume that $\rho:[a, b] \rightarrow \mathbb{R}$ is a nonnegative integrable function with $\int_{a}^{b} \rho(t) d t>0$ and $(p, q)$ is a pair of conjugate exponents, that is $1 \leq p, q \leq \infty, 1 / p+1 / q=1$. Then we have

$$
\begin{gathered}
\left|\int_{a}^{b} \rho(t) F(t) d t-\sum_{j=1}^{m} F\left(a_{j}\right) \int_{a}^{b} \rho(t) H_{0 j}(t) d t-\sum_{j=1}^{m} F^{\prime}\left(a_{j}\right) \int_{a}^{b} \rho(t) H_{1 j}(t) d t\right| \\
\leq\left\|F^{(2 m)}\right\|_{p}\left(\int_{a}^{b}\left|\int_{a}^{b} \rho(t) G_{H}^{C 1}(t, s) d t\right|^{q} d s\right)^{1 / q}
\end{gathered}
$$

where

$$
\begin{aligned}
& H_{0 j}(t)=\frac{P_{m}^{2}(t)}{\left(t-a_{j}\right)^{2}\left[P_{m}^{\prime}\left(a_{j}\right)\right]^{2}}\left(1-\frac{P_{m}^{\prime \prime}\left(a_{j}\right)}{P_{m}^{\prime}\left(a_{j}\right)}\left(t-a_{j}\right)\right), \\
& H_{1 j}(t)=\frac{P_{m}^{2}(t)}{\left(t-a_{j}\right)\left[P_{m}^{\prime}\left(a_{j}\right)\right]^{2}}, \\
& P_{m}(t)=\prod_{j=1}^{m}\left(t-a_{j}\right) \text { for } a \leq a_{1}<a_{2} \ldots<a_{m} \leq b
\end{aligned}
$$


and

$$
G_{H}^{C 1}(t, s)=\left\{\begin{array}{l}
\sum_{j=1}^{l} \frac{\left(a_{j}-s\right)^{2 m-1}}{(2 m-1) !} H_{0 j}(t)+\sum_{j=1}^{l} \frac{\left(a_{j}-s\right)^{2 m-2}}{(2 m-2) !} H_{1 j}(t), s \leq t \\
-\sum_{j=l+1}^{m} \frac{\left(a_{j}-s\right)^{2 m-1}}{(2 m-1) !} H_{0 j}(t)-\sum_{j=l+1}^{m} \frac{\left(a_{j}-s\right)^{2 m-2}}{(2 m-2) !} H_{1 j}(t), s \geq t
\end{array}\right.
$$

for all $a_{l} \leq s \leq a_{l+1}, l=0, \ldots, m\left(a_{0}=a, a_{m+1}=b\right)$. The constant on the right-hand side of (2.4) is sharp for $1<p \leq \infty$ and the best possible for $p=1$.

Remark 1. If we choose $P_{m}$ such that it is orthogonal with weight $\rho(t)$ to all polynomials of lower degree, i.e.

$$
\int_{a}^{b} \rho(t) P_{m}(t) t^{k} d t=0, k=0,1, \ldots, m-1,
$$

we get that

$$
\int_{a}^{b} \rho(t) H_{1 j}(t) d t=0
$$

Now, $a_{1}, a_{2}, \ldots, a_{m}$ are zeros of the orthogonal polynomial $P_{m}$, and using the relation for coefficient $H_{1 j}(t)$, we get

$$
\int_{a}^{b} \rho(t) H_{0 j}(t) d t=\int_{a}^{b} \frac{\rho(t) P_{m}^{2}(t)}{\left(t-a_{j}\right)^{2}\left[P_{m}^{\prime}\left(a_{j}\right)\right]^{2}} d t-\frac{P_{m}^{\prime \prime}\left(a_{j}\right)}{P_{m}^{\prime}\left(a_{j}\right)} \int_{a}^{b} \rho(t) H_{1 j}(t) d t .
$$

Now, using (2.5), we have

$$
\int_{a}^{b} \frac{\rho(t) P_{m}(t)}{\left(t-a_{j}\right) P_{m}^{\prime}\left(a_{j}\right)}\left(\frac{P_{m}(t)}{\left(t-a_{j}\right) P_{m}^{\prime}\left(a_{j}\right)}-1\right) d t=0
$$

because

$$
\frac{P_{m}(t)}{\left(t-a_{j}\right) P_{m}^{\prime}\left(a_{j}\right)}-1=\left(t-a_{j}\right) Q(t)
$$

where $Q(t)$ is polynomial of degree $m-2$. So,

$$
\int_{a}^{b} \rho(t) H_{0 j}(t) d t=\int_{a}^{b} \frac{\rho(t) P_{m}^{2}(t)}{\left(t-a_{j}\right)^{2}\left[P_{m}^{\prime}\left(a_{j}\right)\right]^{2}} d t=\int_{a}^{b} \frac{\rho(t) P_{m}(t)}{\left(t-a_{j}\right) P_{m}^{\prime}\left(a_{j}\right)} d t .
$$

Now, we get inequality

$$
\left|\int_{a}^{b} \rho(t) F(t) d t-\sum_{j=1}^{m} \alpha_{j} F\left(a_{j}\right)\right| \leq\left\|F^{(2 m)}\right\|_{p}\left(\int_{a}^{b}\left|\int_{a}^{b} \rho(t) G_{H}^{C 1}(t, s) d t\right|^{q} d s\right)^{1 / q},
$$

where

$$
\alpha_{j}=\frac{1}{P_{m}^{\prime}\left(a_{j}\right)} \int_{a}^{b} \frac{\rho(t) P_{m}(t)}{\left(t-a_{j}\right)} d t
$$

and for $a_{l} \leq s \leq a_{l+1}$, using the following identity (see [1])

$$
\frac{1}{(2 m-1) !}(t-s)^{(2 m-1)}=\sum_{j=1}^{m} \sum_{i=0}^{k_{j}} \frac{\left(a_{j}-s\right)^{2 m-i-1}}{(2 m-i-1) !} H_{i j}(t)
$$


we get

$\int_{a}^{b} \rho(t) G_{H}^{C 1}(t, s) d t=-\sum_{j=l+1}^{m} \frac{\left(a_{j}-s\right)^{2 m-1} \alpha_{j}}{(2 m-1) !}+\frac{1}{(2 m-1) !} \int_{s}^{b} \rho(t)(t-s)^{2 m-1} d t$.

Corollary 2. Let $\rho(t)=1$ and for $m \geq 1$ the polynomial $P_{m}$ be defined by the formulae

$$
P_{m}(t)=\left|\begin{array}{cccc}
1 & 1 & \cdots & \frac{1}{m} \\
t & \frac{1}{2} & \cdots & \frac{1}{m+1} \\
\vdots & \vdots & \ddots & \vdots \\
t^{m} & \frac{1}{m+1} & \cdots & \frac{1}{2 m}
\end{array}\right|
$$

Then, the orthogonal polynomial $P_{m}$ has $m$ pairwise distinct zeros $\lambda_{1}, \ldots, \lambda_{m}$ in $[0,1]$. Define the coefficients $\alpha_{1}, \ldots, \alpha_{m}$ by (see [4]):

$$
\alpha_{j}=\int_{0}^{1} \frac{P_{m}(t)}{\left(t-\lambda_{j}\right) P_{m}^{\prime}\left(\lambda_{j}\right)} d t
$$

If $f \in C^{2 m}([a, b])$, then it satisfies

$$
\begin{aligned}
& \left|\frac{1}{b-a} \int_{a}^{b} f(t) d t-\sum_{j=1}^{m} \alpha_{j} f\left(\left(1-\lambda_{j}\right) a+\lambda_{j} b\right)\right| \\
\leq & (b-a)^{2 m-1}\left\|f^{(2 m)}\right\|_{p}\left(\int_{a}^{b}\left|\int_{0}^{1} G_{H}^{C 1}\left(t, \frac{s-a}{b-a}\right) d t\right|^{q} d s\right)^{1 / q},
\end{aligned}
$$

where for $\lambda_{l} \leq s \leq \lambda_{l+1}$

$$
\int_{0}^{1} G_{H}^{C 1}(t, s) d t=-\sum_{j=l+1}^{m} \frac{\left(\lambda_{j}-s\right)^{2 m-1} \alpha_{j}}{(2 m-1) !}+\frac{1}{(2 m-1) !} \int_{s}^{1}(t-s)^{2 m-1} d t .
$$

The constant on the right-hand side of inequality is sharp for $1<p \leq \infty$ and the best possible for $p=1$.

Proof. Substitute $a=0, b=1$ and $\rho=1$ into Corollary 1 . Then $P_{m}$ is orthogonal polynomial with respect to the weight function $\rho(t)=1$ since first and $(k+1)$ st columns of the determinant $\left\langle P_{m}(t), t^{k-1}\right\rangle, k=1, \ldots, m$ are linearly dependent. Therefore, $P_{m}$ has $m$ pairwise distinct zeros $0 \leq \lambda_{1}<\cdots<\lambda_{m} \leq 1$. To complete the proof, we apply Remark 1 on the function $F:[0,1] \rightarrow \mathbb{R}$ defined by the formula $F(t)=f((1-t) a+t b)$.

Remark 2. If we put $q=1$, then for $m=1$ in the above corollary we get the midpoint formula (see [8], [14], [16] and [17]). For $m=2$ we get the Gauss 2-point formula (see [11], [14], [16] and [17]). For $m=3$ we get the Gauss 3 -point formula (see [11], [14] and [16]). For $m=4$ we get the Gauss 4-point formula (see [10], [14] and [16]). 
Taking $a_{1}=a$ in Theorem 2 we obtain the following corollaries:

Corollary 3. Suppose that all assumptions of Theorem 1 hold. Assume that $\rho:[a, b] \rightarrow \mathbb{R}$ is a nonnegative integrable function with $\int_{a}^{b} \rho(t) d t>0$ and $(p, q)$ is a pair of conjugate exponents, that is $1 \leq p, q \leq \infty, 1 / p+1 / q=1$. Then we have

$$
\begin{aligned}
& \mid \int_{a}^{b} \rho(t) F(t) d t-\sum_{i=0}^{k_{1}} F^{(i)}(a) \int_{a}^{b} \rho(t) H_{i 1}(t) d t-\sum_{j=2}^{r} \sum_{i=0}^{k_{j}} F^{(i)}\left(a_{j}\right) \\
& \quad \times \int_{a}^{b} \rho(t) H_{i j}(t) d t \mid \leq\left\|F^{(n)}\right\|_{p}\left(\int_{a}^{b}\left|\int_{a}^{b} \rho(t) G_{H}(t, s) d t\right|^{q} d s\right)^{1 / q} .
\end{aligned}
$$

The constant on the right-hand side of (2.7) is sharp for $1<p \leq \infty$ and the best possible for $p=1$.

Corollary 4. Let $F \in C^{2 r-1}([a, b]), r \geq 2$. Assume that $\rho:[a, b] \rightarrow \mathbb{R}$ is a nonnegative integrable function with $\int_{a}^{b} \rho(t) d t>0$ and $(p, q)$ is a pair of conjugate exponents, that is $1 \leq p, q \leq \infty, 1 / p+1 / q=1$. Then we have

$$
\begin{aligned}
& \mid \int_{a}^{b} \rho(t) F(t) d t-F(a) \int_{a}^{b} \rho(t) H_{01}(t) d t \\
& \quad-\sum_{j=2}^{r} F\left(a_{j}\right) \int_{a}^{b} \rho(t) H_{0 j}(t) d t-\sum_{j=2}^{r} F^{\prime}\left(a_{j}\right) \int_{a}^{b} \rho(t) H_{1 j}(t) d t \mid \\
& \quad \leq\left\|F^{(2 r-1)}\right\|_{p}\left(\int_{a}^{b}\left|\int_{a}^{b} \rho(t) G_{H}^{C 4}(t, s) d t\right|^{q} d s\right)^{1 / q}
\end{aligned}
$$

where

$$
\begin{aligned}
H_{01}(t) & =\frac{P_{r-1}^{2}(t)}{P_{r-1}^{2}(a)}, \quad H_{0 j}(t)=\frac{(t-a) P_{r-1}^{2}(t)}{\left(t-a_{j}\right)^{2}\left[P_{r-1}^{\prime}\left(a_{j}\right)\right]^{2}\left(a_{j}-a\right)} \\
\times & \left(1-\frac{P_{r-1}^{\prime}\left(a_{j}\right)+\left(a_{j}-a\right) P_{r-1}^{\prime \prime}\left(a_{j}\right)}{\left(a_{j}-a\right) P_{r-1}^{\prime}\left(a_{j}\right)}\left(t-a_{j}\right)\right), \\
H_{1 j}(t) & =\frac{(t-a) P_{r-1}^{2}(t)}{\left(t-a_{j}\right)\left(a_{j}-a\right)\left[P_{r-1}^{\prime}\left(a_{j}\right)\right]^{2}}, \\
P_{r-1}(t) & =\prod_{j=2}^{r}\left(t-a_{j}\right) \text { for } a=a_{1}<a_{2} \ldots<a_{r} \leq b
\end{aligned}
$$

and

$$
G_{H}^{C 4}(t, s)=\left\{\begin{aligned}
& \frac{(a-s)^{2 r-2}}{(2 r-2) !} H_{01}(t)+\sum_{j=2}^{l} \frac{\left(a_{j}-s\right)^{2 r-2}}{(2 r-2) !} H_{0 j}(t) \\
&+\sum_{j=2}^{l} \frac{\left(a_{j}-s\right)^{2 r-3}}{(2 r-3) !} H_{1 j}(t), s \leq t, \\
&-\sum_{j=l+1}^{r} \frac{\left(a_{j}-s\right)^{2 r-2}}{(2 r-2) !} H_{0 j}(t)-\sum_{j=l+1}^{r} \frac{\left(a_{j}-s\right)^{2 r-3}}{(2 r-3) !} H_{1 j}(t), s \geq t,
\end{aligned}\right.
$$

for all $a_{l} \leq s \leq a_{l+1}, l=1, \ldots, r\left(a_{r+1}=b\right)$. The constant on the right-hand side of (2.8) is sharp for $1<p \leq \infty$ and the best possible for $p=1$. 
Proof. We put $k_{1}=0$ and $k_{j}=1$ for $j=2, \ldots, r$ in Corollary 3.

Remark 3. If we choose $P_{r-1}$ such that it is orthogonal with weight $(t-a) \rho(t)$ to all polynomials of lower degree, i.e.

$$
\int_{a}^{b}(t-a) \rho(t) P_{r-1}(t) t^{k} d t=0, k=0,1, \ldots, r-2
$$

we get that

$$
\int_{a}^{b} \rho(t) H_{1 j}(t) d t=0
$$

Now, similar as in Remark $2, a_{2}, a_{3} \ldots, a_{r}$ are zeros of the orthogonal polynomial $P_{r-1}$, and we get

$$
\int_{a}^{b} \rho(t) H_{0 j}(t) d t=\frac{1}{\left(a_{j}-a\right) P_{r-1}^{\prime}\left(a_{j}\right)} \int_{a}^{b} \frac{\rho(t)(t-a) P_{r-1}(t)}{\left(t-a_{j}\right)} d t .
$$

So,

$$
\begin{aligned}
& \left|\int_{a}^{b} \rho(t) F(t) d t-\alpha_{1} F(a)-\sum_{j=2}^{r} \alpha_{j} F\left(a_{j}\right)\right| \\
& \quad \leq\left\|F^{(2 r-1)}\right\|_{p}\left(\int_{a}^{b}\left|\int_{a}^{b} \rho(t) G_{H}^{C 4}(t, s) d t\right|^{q} d s\right)^{1 / q},
\end{aligned}
$$

where

$$
\alpha_{1}=\frac{1}{P_{r-1}^{2}(a)} \int_{a}^{b} \rho(t) P_{r-1}^{2}(t) d t, \alpha_{j}=\frac{1}{\left(a_{j}-a\right) P_{r-1}^{\prime}\left(a_{j}\right)} \int_{a}^{b} \frac{\rho(t)(t-a) P_{r-1}(t)}{\left(t-a_{j}\right)} d t
$$

and for $a_{l} \leq s \leq a_{l+1}$, using the identity (2.6), we get

$$
\int_{a}^{b} \rho(t) G_{H}^{C 4}(t, s) d t=-\sum_{j=l+1}^{r} \frac{\left(a_{j}-s\right)^{2 r-2} \alpha_{j}}{(2 r-2) !}+\frac{1}{(2 r-2) !} \int_{s}^{b} \rho(t)(t-s)^{2 r-2} d t .
$$

Corollary 5. Let $\rho(t)=1$ and for $r \geq 2$ the polynomial $P_{r-1}$ be defined by the formulae

$$
P_{r-1}(t)=\left|\begin{array}{cccc}
1 & \frac{1}{2} & \cdots & \frac{1}{r} \\
t & \frac{1}{3} & \cdots & \frac{1}{r+1} \\
\vdots & \vdots & \ddots & \vdots \\
t^{r-1} & \frac{1}{r+1} & \cdots & \frac{1}{2 r-1}
\end{array}\right|
$$

Then, the orthogonal polynomial $P_{r-1}$ has $r-1$ distinct zeros $\lambda_{2}, \ldots, \lambda_{r}$ in $[0,1]$. Define the coefficients $\alpha_{1}, \ldots, \alpha_{r}$ by (see [4])

$$
\alpha_{1}=\frac{1}{P_{r-1}^{2}(0)} \int_{0}^{1} P_{r-1}^{2}(t) d t, \quad \alpha_{j}=\frac{1}{\lambda_{j}} \int_{0}^{1} \frac{t P_{r-1}(t)}{\left(t-\lambda_{j}\right) P_{r-1}^{\prime}\left(\lambda_{j}\right)} d t .
$$


If $f \in C^{2 r-1}([a, b])$, then it satisfies

$$
\begin{aligned}
& \left|\frac{1}{b-a} \int_{a}^{b} f(t) d t-\alpha_{1} f(a)-\sum_{j=2}^{r} \alpha_{j} f\left(\left(1-\lambda_{j}\right) a+\lambda_{j} b\right)\right| \\
& \quad \leq(b-a)^{2 r-2}\left\|f^{(2 r-1)}\right\|_{p}\left(\int_{a}^{b}\left|\int_{0}^{1} G_{H}^{C 4}\left(t, \frac{s-a}{b-a}\right) d t\right|^{q} d s\right)^{1 / q}
\end{aligned}
$$

where for $a_{l} \leq s \leq a_{l+1}$

$$
\int_{0}^{1} G_{H}^{C 4}(t, s) d t=-\sum_{j=l+1}^{r} \frac{\left(\lambda_{j}-s\right)^{2 r-2} \alpha_{j}}{(2 r-2) !}+\frac{1}{(2 r-2) !} \int_{s}^{1}(t-s)^{2 r-2} d t .
$$

The constant on the right-hand side of inequality is sharp for $1<p \leq \infty$ and the best possible for $p=1$.

Proof. Substitute $a=0, b=1$ and $\rho=1$ into Corollary 4 . Then $P_{r-1}$ is orthogonal polynomial with respect to the weight function $\rho(t)=t$. The rest of proof is similar to the proof of Corollary 2.

Remark 4. If we put $q=1$, then for $r=2$ in the above corollary we get the Radau 2-point formula (see [9], [14] and [16]). For $r=3$ we get the Radau 3 -point formula (see [9], [14] and [16]).

Remark 5. For $a_{r}=b$ in Theorem 2 we obtain the similar results as above.

Taking $a_{1}=a, a_{r}=b$ in Theorem 2 we obtain the following corollaries:

Corollary 6. Suppose that all assumptions of Theorem 1 hold. Assume that $\rho:[a, b] \rightarrow \mathbb{R}$ is a nonnegative integrable function with $\int_{a}^{b} \rho(t) d t>0$ and $(p, q)$ is a pair of conjugate exponents, that is $1 \leq p, q \leq \infty, 1 / p+1 / q=1$. Then we have

$$
\begin{aligned}
& \mid \int_{a}^{b} \rho(t) F(t) d t-\sum_{i=0}^{k_{1}} F^{(i)}(a) \int_{a}^{b} \rho(t) H_{i 1}(t) d t \\
& \quad-\sum_{j=2}^{r-1} \sum_{i=0}^{k_{j}} F^{(i)}\left(a_{j}\right) \int_{a}^{b} \rho(t) H_{i j}(t) d t-\sum_{i=0}^{k_{r}} F^{(i)}(b) \int_{a}^{b} \rho(t) H_{i r}(t) d t \mid \\
& \leq\left\|F^{(n)}\right\|_{p}\left(\int_{a}^{b}\left|\int_{a}^{b} \rho(t) G_{H}(t, s) d t\right|^{q} d s\right)^{1 / q}
\end{aligned}
$$

The constant on the right-hand side of (2.9) is sharp for $1<p \leq \infty$ and the best possible for $p=1$.

Corollary 7. Let $F \in C^{2 r-2}([a, b]), r \geq 2$. Assume that $\rho:[a, b] \rightarrow \mathbb{R}$ is a nonnegative integrable function with $\int_{a}^{b} \rho(t) d t>0$ and $(p, q)$ is a pair of conjugate 
exponents, that is $1 \leq p, q \leq \infty, 1 / p+1 / q=1$. Then we have

$$
\begin{gathered}
\mid \int_{a}^{b} \rho(t) F(t) d t-F(a) \int_{a}^{b} \rho(t) H_{01}(t) d t-\sum_{j=2}^{r-1} F\left(a_{j}\right) \int_{a}^{b} \rho(t) H_{0 j}(t) d t \\
-\sum_{j=2}^{r-1} F^{\prime}\left(a_{j}\right) \int_{a}^{b} \rho(t) H_{1 j}(t) d t-F(b) \int_{a}^{b} \rho(t) H_{0 r}(t) d t \mid \\
\leq\left\|F^{(2 r-2)}\right\|_{p}\left(\int_{a}^{b}\left|\int_{a}^{b} \rho(t) G_{H}^{C 7}(t, s) d t\right|^{q} d s\right)^{1 / q}
\end{gathered}
$$

where

$$
\begin{aligned}
& H_{01}(t)=\frac{(b-t) P_{r-2}^{2}(t)}{(b-a) P_{r-2}^{2}(a)}, \quad H_{0 r}(t)=\frac{(t-a) P_{r-2}^{2}(t)}{(b-a) P_{r-2}^{2}(b)}, \\
& H_{0 j}(t)=\frac{(t-a)(b-t) P_{r-2}^{2}(t)}{\left(a_{j}-a\right)\left(b-a_{j}\right)\left(t-a_{j}\right)^{2}\left[P_{r-2}^{\prime}\left(a_{j}\right)\right]^{2}} \\
& \quad \times\left(1+\frac{\left(2 a_{j}-a-b\right) P_{r-2}^{\prime}\left(a_{j}\right)-\left(b-a_{j}\right)\left(a_{j}-a\right) P_{r-2}^{\prime \prime}\left(a_{j}\right)}{\left(b-a_{j}\right)\left(a_{j}-a\right) P_{r-2}^{\prime}\left(a_{j}\right)}\left(t-a_{j}\right)\right), \\
& H_{1 j}(t)=\frac{(t-a)(b-t) P_{r-2}^{2}(t)}{\left(t-a_{j}\right)\left(a_{j}-a\right)\left(b-a_{j}\right)\left[P_{r-2}^{\prime}\left(a_{j}\right)\right]^{2}}, \\
& P_{r-2}(t)=\prod_{j=2}^{r-1}\left(t-a_{j}\right) \text { for } a=a_{1}<a_{2}<\ldots<a_{r-1}<a_{r}=b
\end{aligned}
$$

and

$$
G_{H}^{C 7}(t, s)=\left\{\begin{array}{c}
\frac{(a-s)^{2 r-3}}{(2 r-3) !} H_{01}(t)+\sum_{j=2}^{l} \frac{\left(a_{j}-s\right)^{2 r-3}}{(2 r-3) !} H_{0 j}(t) \\
\quad+\sum_{j=2}^{l} \frac{\left(a_{j}-s\right)^{2 r-4}}{(2 r-4) !} H_{1 j}(t), s \leq t, \\
-\sum_{j=l+1}^{r-1} \frac{\left(a_{j}-s\right)^{2 r-3}}{(2 r-3) !} H_{0 j}(t)-\sum_{j=l+1}^{r-1} \frac{\left(a_{j}-s\right)^{2 r-4}}{(2 r-4) !} H_{1 j}(t) \\
-\frac{(b-s)^{2 r-3}}{(2 r-3) !} H_{0 r}(t), s \geq t,
\end{array}\right.
$$

for all $a_{l} \leq s \leq a_{l+1}, l=1, \ldots, r-1$. The constant on the right-hand side of (2.10) is sharp for $1<p \leq \infty$ and the best possible for $p=1$.

Proof. We put $k_{1}=k_{r}=0$ and $k_{j}=1$ for $j=2, \ldots, r-1$ in Corollary 6.

Remark 6 . If we choose $P_{r-2}$ such that it is orthogonal with weight $(t-a)(b-$ $t) \rho(t)$, to all polynomials of lower degree, i.e.

$$
\int_{a}^{b} \rho(t)(t-a)(b-t) P_{r-2}(t) t^{l} d t=0, \quad l=0,1, \ldots, r-3,
$$

we get that

$$
\int_{a}^{b} \rho(t) H_{1 j}(t) d t=0
$$


and then similar as in Remark $2, a_{2}, a_{3}, \ldots, a_{r-1}$ are zeros of the orthogonal polynomial $P_{r-2}$, and

$$
\int_{a}^{b} \rho(t) H_{0 j}(t) d t=\frac{1}{\left(a_{j}-a\right)\left(b-a_{j}\right) P_{r-2}^{\prime}\left(a_{j}\right)} \int_{a}^{b} \frac{\rho(t)(t-a)(b-t) P_{r-2}(t)}{\left(t-a_{j}\right)} d t .
$$

So,

$$
\begin{gathered}
\left|\int_{a}^{b} \rho(t) F(t) d t-\alpha_{1} F(a)-\sum_{j=2}^{r-1} \alpha_{j} F\left(a_{j}\right)-\alpha_{r} F(b)\right| \\
\quad \leq\left\|F^{(2 r-2)}\right\|_{p}\left(\int_{a}^{b}\left|\int_{a}^{b} \rho(t) G_{H}(t, s) d t\right|^{q} d s\right)^{1 / q}
\end{gathered}
$$

where

$$
\begin{aligned}
\alpha_{1} & =\frac{1}{(b-a) P_{r-2}^{2}(a)} \int_{a}^{b} \rho(t)(b-t) P_{r-2}^{2}(t) d t \\
\alpha_{j} & =\frac{1}{\left(a_{j}-a\right)\left(b-a_{j}\right) P_{r-2}^{\prime}\left(a_{j}\right)} \int_{a}^{b} \frac{\rho(t)(t-a)(b-t) P_{r-2}(t)}{\left(t-a_{j}\right)} d t \\
\alpha_{r} & =\frac{1}{(b-a) P_{r-2}^{2}(b)} \int_{a}^{b} \rho(t)(t-a) P_{r-2}^{2}(t) d t
\end{aligned}
$$

and for $a_{l} \leq s \leq a_{l+1}$, using the identity (2.6), we get

$$
\int_{a}^{b} \rho(t) G_{H}^{C 7}(t, s) d t=-\sum_{j=l+1}^{r} \frac{\left(a_{j}-s\right)^{2 r-3} \alpha_{j}}{(2 r-3) !}+\frac{1}{(2 r-3) !} \int_{s}^{b} \rho(t)(t-s)^{2 r-3} d t .
$$

Corollary 8 . Let $\rho(t)=1$ and for $r \geq 3$ the polynomial $P_{r-2}$ be defined by the formulae

$$
P_{r-2}(t)=\left|\begin{array}{cccc}
1 & \frac{1}{2 \cdot 3} & \cdots & \frac{1}{(r-1) r} \\
t & \frac{1}{3 \cdot 4} & \cdots & \frac{1}{r(r+1)} \\
\vdots & \vdots & \ddots & \vdots \\
t^{r-2} & \frac{1}{r(r+1)} & \cdots & \frac{1}{(2 r-3)(2 r-2)}
\end{array}\right|
$$

Then, the orthogonal polynomial $P_{r-2}$ has $r-2$ distinct zeros $\lambda_{2}, \ldots, \lambda_{r-1}$ in $[0,1]$. Define the coefficients $\alpha_{1}, \ldots, \alpha_{r}$ by (see [4]):

$$
\begin{aligned}
\alpha_{1} & =\frac{1}{P_{r-2}^{2}(0)} \int_{0}^{1}(1-t) P_{r-2}^{2}(t) d t, \quad \alpha_{r}=\frac{1}{P_{r-2}^{2}(1)} \int_{0}^{1} t P_{r-2}^{2}(t) d t \\
\alpha_{j} & =\frac{1}{\left(1-\lambda_{j}\right) \lambda_{j}} \int_{0}^{1} \frac{t(1-t) P_{r-2}(t)}{\left(t-\lambda_{j}\right) P_{r-2}^{\prime}\left(\lambda_{j}\right)} d t .
\end{aligned}
$$

If $f \in C^{2 r-2}([a, b])$, then it satisfies

$$
\begin{aligned}
& \left|\frac{1}{b-a} \int_{a}^{b} f(t) d t-\alpha_{1} f(a)-\sum_{j=2}^{r-1} \alpha_{j} f\left(\left(1-\lambda_{j}\right) a+\lambda_{j} b\right)-\alpha_{r} f(b)\right| \\
& \quad \leq(b-a)^{2 r-3}\left\|f^{(2 r-2)}\right\|_{p}\left(\int_{a}^{b}\left|\int_{0}^{1} G_{H}^{C 7}\left(t, \frac{s-a}{b-a}\right) d t\right|^{q} d s\right)^{1 / q}
\end{aligned}
$$


where

$$
\int_{0}^{1} G_{H}^{C 7}(t, s) d t=-\sum_{j=l+1}^{r} \frac{\left(\lambda_{j}-s\right)^{2 r-3} \alpha_{j}}{(2 r-3) !}+\frac{1}{(2 r-3) !} \int_{s}^{1}(t-s)^{2 r-3} d t .
$$

The constant on the right-hand side of inequality is sharp for $1<p \leq \infty$ and the best possible for $p=1$.

Proof. Substitute $a=0, b=1$ and $\rho=1$ into Corollary 7. Then $P_{r-2}$ is orthogonal polynomial with respect to the weight function $\rho(t)=(1-t) t$. The rest of proof is similar to the proof of Corollary 2 .

Remark \%. If we put $q=1$, then for $r=2$ in the above corollary we get the trapezoid formula (see [7], [14], [16] and [17]). For $r=3$ we get the Simpson formula (see [6], [15], [14] and [16]). For $r=4$ we get the Lobatto 4-point formula (see [12], [14] and [16]). For $r=5$ we get the Lobatto 5-point formula (see [13], [14] and [16]).

Taking $r=2,1 \leq m \leq n-1, k_{1}=m-1, k_{2}=n-m-1$ in Corollary 6 we obtain the inequalities with $(m, n-m)$ type conditions:

Corollary 9. Suppose that all assumptions of Theorem 1 hold. Assume that $\rho:[a, b] \rightarrow \mathbb{R}$ is a nonnegative integrable function with $\int_{a}^{b} \rho(t) d t>0$ and $(p, q)$ is a pair of conjugate exponents, that is $1 \leq p, q \leq \infty, 1 / p+1 / q=1$. Then we have

$$
\begin{aligned}
& \mid \int_{a}^{b} \rho(t) F(t) d t-\sum_{i=0}^{m-1} F^{(i)}(a) \int_{a}^{b} \rho(t) H_{i 1}(t) d t-\sum_{i=0}^{n-m-1} F^{(i)}(b) \\
& \quad \times \int_{a}^{b} \rho(t) H_{i 2}(t) d t \mid \leq\left\|F^{(n)}\right\|_{p}\left(\int_{a}^{b}\left|\int_{a}^{b} \rho(t) G_{H}(t, s) d t\right|^{q} d s\right)^{1 / q} .
\end{aligned}
$$

The constant on the right-hand side of (2.11) is sharp for $1<p \leq \infty$ and the best possible for $p=1$.

Taking $n=2 m, r=2, k_{1}=k_{2}=m-1$ in Corollary 6 we obtain the inequalities with two-point Taylor conditions:

Corollary 10. Suppose that all assumptions of Theorem 1 hold. Assume that $\rho:[a, b] \rightarrow \mathbb{R}$ is a nonnegative integrable function with $\int_{a}^{b} \rho(t) d t>0$ and $(p, q)$ is a pair of conjugate exponents, that is $1 \leq p, q \leq \infty, 1 / p+1 / q=1$. Then we have

$$
\begin{aligned}
& \mid \int_{a}^{b} \rho(t) F(t) d t-\sum_{i=0}^{m-1} F^{(i)}(a) \int_{a}^{b} \rho(t) H_{i 1}(t) d t-\sum_{i=0}^{m-1} F^{(i)}(b) \\
& \quad \times \int_{a}^{b} \rho(t) H_{i 2}(t) d t \mid \leq\left\|F^{(2 m)}\right\|_{p}\left(\int_{a}^{b}\left|\int_{a}^{b} \rho(t) G_{H}(t, s) d t\right|^{q} d s\right)^{1 / q}
\end{aligned}
$$


where

$$
\begin{aligned}
& H_{i 1}(t)=\frac{(t-a)^{i}(t-b)^{m}}{i !} \sum_{k=0}^{m-1-i} \frac{(-1)^{k}(m+k-1) !}{[(m-1) !]^{2}(a-b)^{m+k}}(t-a)^{k}, \\
& H_{i 2}(t)=\frac{(t-a)^{m}(t-b)^{i}}{i !} \sum_{k=0}^{m-1-i} \frac{(-1)^{k}(m+k-1) !}{[(m-1) !]^{2}(b-a)^{m+k}}(t-b)^{k} .
\end{aligned}
$$

The constant on the right-hand side of (2.12) is sharp for $1<p \leq \infty$ and the best possible for $p=1$.

\section{Inequalities related to the bounds for the Čebyšev functional}

For two Lebesgue integrable functions $f, h:[a, b] \rightarrow \mathbb{R}$ we consider Čebyšev functional

$$
\Omega(f, h)=\frac{1}{b-a} \int_{a}^{b} f(t) h(t) d t-\frac{1}{b-a} \int_{a}^{b} f(t) d t \frac{1}{b-a} \cdot \int_{a}^{b} h(t) d t .
$$

In [5], the authors proved the following theorems:

Theorem 3. Let $f:[a, b] \rightarrow \mathbb{R}$ be a Lebesgue integrable function and $h$ : $[a, b] \rightarrow \mathbb{R}$ be an absolutely continuous function with $x \mapsto(x-a)(b-x)\left[h^{\prime}(x)\right]^{2} \in$ $L[a, b]$. Then we have the inequality

$$
|\Omega(f, h)| \leq \frac{1}{\sqrt{2}}[\Omega(f, f)]^{\frac{1}{2}} \frac{1}{\sqrt{b-a}}\left(\int_{a}^{b}(x-a)(b-x)\left[h^{\prime}(x)\right]^{2} d x\right)^{\frac{1}{2}} .
$$

The constant $\frac{1}{\sqrt{2}}$ in (3.2) is the best possible.

Theorem 4. Assume that $h:[a, b] \rightarrow \mathbb{R}$ is monotonic nondecreasing on $[a, b]$ and $f:[a, b] \rightarrow \mathbb{R}$ is absolutely continuous with $f^{\prime} \in L_{\infty}[a, b]$. Then we have the inequality

$$
|\Omega(f, h)| \leq \frac{1}{2(b-a)}\left\|f^{\prime}\right\|_{\infty} \int_{a}^{b}(x-a)(b-x) d h(x) .
$$

The constant $\frac{1}{2}$ in (3.3) is the best possible.

In this section we obtain some new estimations of the remainder in quadrature formulae of Hermite type by using Theorem 3 and Theorem 4.

Theorem 5. Let $F:[a, b] \rightarrow \mathbb{R}$ be such that $F \in C^{n+1}[a, b]$ for $n \in \mathbb{N}$ and let the function $\Phi$ and functional $\Omega$ be defined in (2.2) and (3.1) respectively. Then

$$
\begin{aligned}
& \int_{a}^{b} \rho(t) F(t) d t-\sum_{j=1}^{r} \sum_{i=0}^{k_{j}} \int_{a}^{b} \rho(t) H_{i j}(t) F^{(i)}\left(a_{j}\right) d t \\
& \quad=\frac{F^{(n-1)}(b)-F^{(n-1)}(a)}{(b-a) n !} \int_{a}^{b} \rho(t) \omega(t) d t+H_{n}^{1}(F ; a, b),
\end{aligned}
$$


where the remainder $H_{n}^{1}(F ; a, b)$ satisfies the estimation

$$
\left|H_{n}^{1}(F ; a, b)\right| \leq \frac{\sqrt{b-a}}{\sqrt{2}}\left[\Omega(\Phi, \Phi]^{\frac{1}{2}}\left|\int_{a}^{b}(s-a)(b-s)\left[F^{(n+1)}(s)\right]^{2} d s\right|^{\frac{1}{2}} .\right.
$$

Proof. Because $F^{(n+1)}$ is continuous function then the function $F^{(n)}$ is absolutely continuous (see Theorem 39.15. from [2]) and we can apply results from Theorem 3 for $f \rightarrow \Phi$ and $h \rightarrow F^{(n)}$. So, we obtain

$$
\begin{aligned}
& \left|\frac{1}{b-a} \int_{a}^{b} \Phi(s) F^{(n)}(s) d s-\frac{1}{b-a} \int_{a}^{b} \Phi(s) d s \frac{1}{b-a} \int_{a}^{b} F^{(n)}(s) d s\right| \\
& \leq \frac{1}{\sqrt{2}}[\Omega(\Phi, \Phi)]^{\frac{1}{2}} \frac{1}{\sqrt{b-a}}\left|\int_{a}^{b}(s-a)(b-s)\left[F^{(n+1)}(s)\right]^{2} d s\right|^{\frac{1}{2}} .
\end{aligned}
$$

Therefore we have

$$
\int_{a}^{b} \Phi(s) F^{(n)}(s) d s=\frac{F^{(n-1)}(b)-F^{(n-1)}(a)}{b-a} \int_{a}^{b} \rho(t) d t \int_{a}^{b} G_{H}(t, s) d s+H_{n}^{1}(F ; a, b),
$$

where the remainder $H_{n}^{1}(F ; a, b)$ satisfies estimation (3.5). Now from identity (1.1) (see [1, pg.76]) we obtain (3.4).

Using Theorem 4 we obtain the following Grüss type inequality.

Theorem 6. Let $F:[a, b] \rightarrow \mathbb{R}$ be such that $F \in C^{n+1}[a, b]$ for $n \in \mathbb{N}$ and $F^{(n+1)} \geq 0$ on $[a, b]$ and let the function $\Phi$ be defined in (2.2). Then we have the representation (3.4) and the remainder $H_{n}^{1}(F ; a, b)$ satisfies the bound

$$
\begin{gathered}
\left|H_{n}^{1}(F ; a, b)\right| \leq\left\|\Phi^{\prime}\right\|_{\infty}\left\{\frac{(b-a)\left[F^{(n-1)}(b)+F^{(n-1)}(a)\right]}{2}\right. \\
\left.-\left[F^{(n-2)}(b)-F^{(n-2)}(a)\right]\right\} .
\end{gathered}
$$

Proof. Applying Theorem 4 for $f \rightarrow \Phi$ and $h \rightarrow F^{(n)}$ we obtain

$$
\begin{aligned}
& \left|\frac{1}{b-a} \int_{a}^{b} \Phi(s) F^{(n)}(s) d s-\frac{1}{b-a} \int_{a}^{b} \Phi(s) d s \frac{1}{b-a} \int_{a}^{b} F^{(n)}(s) d s\right| \\
& \leq \frac{\left\|\Phi^{\prime}\right\|_{\infty}}{2(b-a)} \int_{a}^{b}(s-a)(b-s) F^{(n+1)}(s) d s .
\end{aligned}
$$

Since

$$
\begin{aligned}
\int_{a}^{b}( & s-a)(b-s) F^{(n+1)}(s) d s=\int_{a}^{b}[2 s-(a+b)] F^{(n)}(s) d s \\
& =(b-a)\left[F^{(n-1)}(b)+F^{(n-1)}(a)\right]-2\left[F^{(n-2)}(b)-F^{(n-2)}(a)\right],
\end{aligned}
$$

using identities (1.1) and (3.7) we obtain (3.6)

Remark 8. Similarly as in the second section we can get the special cases of above theorems from different choices of $r, k_{j}$ and $a_{j}$, and also for the zeros of orthogonal polynomials. 


\section{Acknowledgements}

The research of the authors has been fully supported by Croatian Science Foundation under the project 5435 .

\section{References}

[1] R.P. Agarwal and P.J.Y. Wong. Error Inequalities in Polynomial Interpolation and Their Applications. Kluwer Academic Publishers, Dordrecht / Boston / London, 1993. http://dx.doi.org/10.1007/978-94-011-2026-5.

[2] C.D. Aliprantis and O. Burkinshaw. Principles of Real Analysis. Academic Press, San Diego/ London / Boston / New York / Sydney / Tokyo / Toronto, 1998.

[3] G. Aras-Gazić, J. Pečarić and A. Vukelić. Cauchy's error representation of Hermite interpolating polynomial and related results. accepted for publication in Math. Ineq. Appl.

[4] M. Bessenyei and Zs. Páles. Higher-order generalizations of Hadamard's inequality. Publ. Math. Debrecen, 61(3-4):623-643, 2002.

[5] P. Cerone and S.S. Dragomir. Some new Ostrowsky-type bounds for the Cebyšev functional and applications. J. Math. Inequal., 8(1):159-170, 2014. http://dx.doi.org/10.7153/jmi-08-10.

[6] Lj. Dedić, M. Matić and J. Pečarić. On Euler-Simpson formulae. Pan. Amer. Math. J., 11(2):47-64, 2001.

[7] Lj. Dedić, M. Matić and J. Pečarić. On Euler trapezoid formulae. Appl. Math. Comput., 123(1):37-62, 2001. http://dx.doi.org/10.1016/S0096-3003(00)000540 .

[8] Lj. Dedić, M. Matić and J. Pečarić. On Euler midpoint formulae. ANZIAM J, 46(3):417-438, 2005. http://dx.doi.org/10.1017/S144618110000835X.

[9] I. Franjić. Hermite-Hadamard-type inequalities for Radau-type quadrature rules. J. Math. Inequal., 3(3):395-407, 2009. http://dx.doi.org/10.7153/jmi-03-39.

[10] I. Franjić, J. Pečarić and I. Perić. Estimates for the Gauss four-point formula for functions with low degree of smoothness. Appl. Math. Lett., 20(1):1-6, 2007. http://dx.doi.org/10.1016/j.aml.2006.02.013.

[11] I. Franjić, J. Pečarić and I. Perić. Quadrature formulae of Gauss type based on Euler identities. Math. Comput. Modelling, 45(3-4):355-370, 2007. http://dx.doi.org/10.1016/j.mcm.2006.05.009.

[12] I. Franjić, J. Pečarić and I. Perić. General closed 4-point quadrature formulae of Euler type. Math. Inequal.Appl., 12(3):4516-4528, 2009. http://dx.doi.org/10.7153/mia-12-45.

[13] I. Franjić, J. Pečarić and I. Perić. On families of quadrature formulas based on Euler identities. Appl. Math. Comput., 217(9):4516-4528, 2011. http://dx.doi.org/10.1016/j.amc.2010.11.002.

[14] I. Franjić, J. Pečarić, I. Perić and A. Vukelić. Euler integral identity, quadrature formulae and error estimations. Element, Zagreb, 2011.

[15] I. Franjić, J. Pečarić and A. Vukelić. General Euler-Simpson formulae. Indian J. Math., 47(2-3):139-157, 2005. 
[16] V.I. Krylov. Approximate calculation of integrals. Macmillan, New York-London, 1962.

[17] J. Pečarić, I. Perić and A. Vukelić. Sharp integral inequalities based on general Euler two-point formulae. ANZIAM J, 46(4):1-20, 2005. http://dx.doi.org/10.1017/S1446181100009676. 